\title{
Regulation of histone H2A.Z expression is mediated by sirtuin 1 in prostate cancer
}

\author{
Tiago Baptista ${ }^{1,2}$, Inês Graça ${ }^{1,2,4}$, Elsa J. Sousa ${ }^{1,2}$, Ana I. Oliveira ${ }^{1,2}$, Natália R. \\ Costa $^{1,2}$, Pedro Costa-Pinheiro ${ }^{1,2}$, Francisco Amado ${ }^{5}$, Rui Henrique ${ }^{1,3,6}$ and Carmen \\ Jerónimo ${ }^{1,2,6}$ \\ ${ }^{1}$ Cancer Epigenetics Group, Research Center, Portuguese Oncology Institute - Porto, Portugal \\ 2 Department of Genetics, Portuguese Oncology Institute - Porto, Portugal \\ 3 Department of Pathology, Portuguese Oncology Institute - Porto, Portugal \\ ${ }^{4}$ School of Allied Health Sciences ESTSP, Polytechnic of Porto \\ ${ }^{5}$ Department of Chemistry, Universidade de Aveiro, Campus Universitário de Santiago, Aveiro, Portugal \\ ${ }^{6}$ Department of Pathology and Molecular Immunology, Institute of Biomedical Sciences Abel Salazar, University of Porto, \\ Portugal \\ Correspondence to: Carmen Jerónimo, email: carmenjeronimo@ipoporto.min-saude.pt \\ Keywords: Prostate cancer, H2A.Z, sirtuin 1, epigenetic drugs \\ Received: August 1, $2013 \quad$ Accepted: August 27, $2013 \quad$ Published: August 29, 2013
}

This is an open-access article distributed under the terms of the Creative Commons Attribution License, which permits unrestricted use, distribution, and reproduction in any medium, provided the original author and source are credited.

\section{ABSTRACT:}

Histone variants seem to play a major role in gene expression regulation. In prostate cancer, H2A.Z and its acetylated form are implicated in oncogenes' upregulation. SIRT1, which may act either as tumor suppressor or oncogene, reduces H2A.Z levels in cardiomyocytes, via proteasome-mediated degradation, and this mechanism might be impaired in prostate cancer cells due to sirtuin 1 downregulation. Thus, we aimed to characterize the mechanisms underlying H2A.Z and SIRT1 deregulation in prostate carcinogenesis and how they interact.

We found that H2AFZ and SIRT1 were up- and downregulated, respectively, at transcript level in primary prostate cancer and high-grade prostatic intraepithelial neoplasia compared to normal prostatic tissues. Induced SIRT1 overexpression in prostate cancer cell lines resulted in almost complete absence of H2A.Z. Inhibition of $m$ TOR had a modest effect on H2A.Z levels, but proteasome inhibition prevented the marked reduction of H2A.Z due to sirtuin 1 overexpression. Prostate cancer cells exposed to epigenetic modifying drugs trichostatin $A$, alone or combined with 5-aza2'-deoxycytidine, increased H2AFZ transcript, although with a concomitant decrease in protein levels. Conversely, SIRT1 transcript and protein levels increased after exposure. ChIP revealed an increase of activation marks within the TSS region for both genes. Remarkably, inhibition of sirtuin 1 with nicotinamide, increased H2A.Z levels, whereas activation of sirtuin 1 by resveratrol led to an abrupt decrease in H2A.Z. Finally, protein-ligation assay showed that exposure to epigenetic modifying drugs fostered the interaction between sirtuin 1 and H2A.Z.

We concluded that sirtuin 1 and H2A.Z deregulation in prostate cancer are reciprocally related. Epigenetic mechanisms, mostly histone post-translational modifications, are likely involved and impair sirtuin 1-mediated downregulation of H2A.Z via proteasome-mediated degradation. Epigenetic modifying drugs in conjunction with enzymatic modulators are able to restore the normal functions of sirtuin 1 and might constitute relevant tools for targeted therapy of prostate cancer patients. 


\section{INTRODUCTION}

Prostate cancer $(\mathrm{PCa})$ is the second most common malignancy diagnosed in men worldwide, being one of the major causes of cancer-related morbidity and mortality[1]. Although mostly symptomatic only in advanced stages, $\mathrm{PCa}$ is characterized by early disruption of both genetic[2] and epigenetic[3] mechanisms, progressively becoming more severe throughout disease progression.

Alterations in histone variants are the least understood among epigenetic mechanisms deregulated in cancer. Histone variant H2A.Z, encoded by $H 2 A F Z$, whose insertion into chromatin is tightly regulated by complexes such as SRCAP, p400/Tip60 and TIP48/49 [4], plays a major role in critical biological processes, such as chromosome segregation [5], cell cycle progression [6] and maintenance of heterochromatin/euchromatin status [7]. Nevertheless, H2A.Z has been consistently associated with neoplastic transformation: $H 2 A F \mathrm{Z}$ overexpression has been reported in sporadic microsatellite unstable colorectal carcinoma [8], breast carcinoma $[9,10]$ and several undifferentiated cancers [11]. At a molecular level, this histone variant is responsible for overexpression of several proto-oncogenes, including $C-M Y C[9,12]$. In $\mathrm{PCa}$, however, the role of H2A.Z remains elusive [13]. Increased expression of $H 2 A F Z$ was found in a castrationresistant xenograph model of $\mathrm{PCa}$, suggesting that high levels of H2A.Z might be predictive to progression for androgen-independent disease [14]. Conversely, it has been claimed that acetylated H2A.Z (acH2A.Z), and not H2A.Z itself, was enriched (oncogenes) or lost (tumor suppressor genes, TSG) at the transcription start-site (TSS) of nucleosomes during carcinogenesis, suggesting that acH2A.Z contributes for gene expression deregulation in $\mathrm{PCa}$ [15].

It has been previously reported that sirtuin 1 , a member of class III histone deacetylases (HDACs), negatively regulates H2A.Z levels in cardiomyocytes, through targeting of this histone variant to degradation via an ubiquitin/proteasome pathway [16]. However, the role of sirtuin 1 in carcinogenesis remains unclear [17, 18]. Indeed, this HDAC is overexpressed in some cancers $[19,20]$, but downregulated in others [21], supporting its role either as an oncogene or a TSG. In PCa, both SIRT1 over- and underexpression have been reported [21, 22]. Nevertheless, there is accumulating evidence that sirtuin 1 mainly acts as a tumor suppressor protein [23-25], due to its ability to promote the activity of $\mathrm{TSC} 2$, a repressor of mTOR [26].

In this study, we aimed to uncover the putative regulatory role of sirtuin 1 in H2A.Z expression during prostate carcinogenesis and determine whether it might constitute a relevant therapeutic target for $\mathrm{PCa}$.

\section{RESULTS}

\section{SIRT1 and H2AFZ are deregulated in PCa}

$H 2 A F Z$ and SIRT1 transcript levels were assessed in primary $\mathrm{PCa}$, as well as in high-grade prostatic intraepithelial neoplasia (PIN) and morphologically normal prostate tissue (NPT). Relevant clinical and histopathological data are depicted in Table 1. No statistically significant differences were found for age between patients and controls (NPT).

Statistically significant differences were observed in SIRT1 and H2AFZ transcript levels among the three analyzed groups. Both PIN lesions and PCa showed downregulation of SIRT1 with concomitant overexpression of $H 2 A F Z$, compared to NPT (Fig. 1A). No statistically significant differences were disclosed for SIRT1 and $H 2 A F Z$ expression levels between PIN and PCa samples, and no associations were found with clinicopathological variables in $\mathrm{PCa}$ patients.

Expression profiling of $\mathrm{PCa}$ cell lines $\mathrm{LNCaP}$, DU145, and PC-3 revealed that SIRT1 and H2AFZ mRNA levels were within the same range as that observed in primary $\mathrm{PCa}$ tissue samples (Fig. 1B).
A

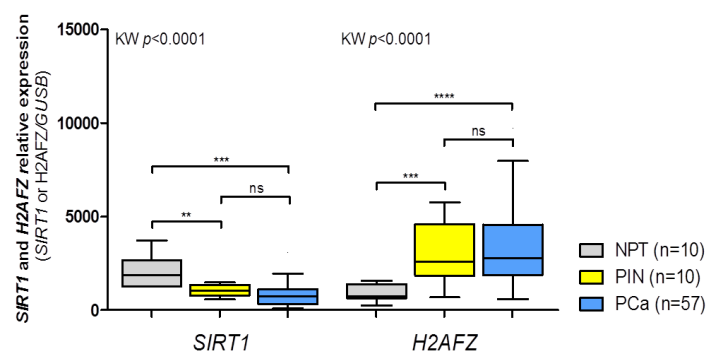

B

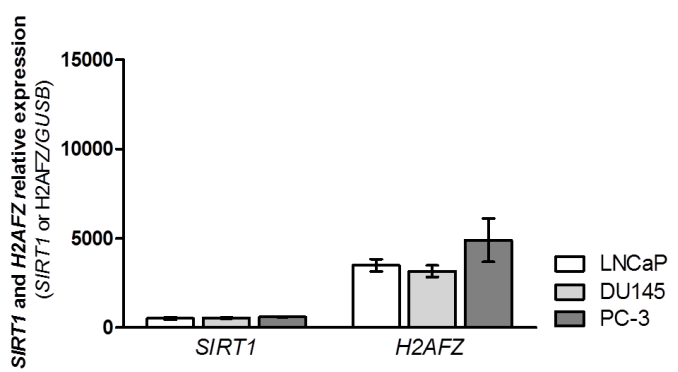

Figure 1: Transcriptional status of SIRT1 and $H 2 A F Z$ in clinical samples (normal prostate tissues - NPT -, prostatic intraepithelial neoplasia - PIN - and prostate carcinoma - PCa) and PCa cell lines (LNCaP, DU145 and PC-3). (A) SIRT1 downregulation and $H 2 A F Z$ overexpression are early events in prostatic carcinogenesis (group analysis with Kruskal-Wallis test followed by a pairwise Mann-Whitney $U$ test, ${ }^{* *} p<0.01,{ }^{* * *} p<0.001$ and $* * * * p<0.0001$ ); (B) SIRT1 and H2AFZ gene expression in PCa cell lines follow the tendency observed for clinical samples of prostatic malignancy (mean $\pm \mathrm{SD}, \mathrm{n}=3$ ). 
Table 1: Clinical and histopathological features of patient populations.

\begin{tabular}{|l|c|c|c|}
\hline Clinicopathological Features & NPT & PIN & PCa \\
\hline Patients, $\mathrm{n}$ & 10 & 10 & 57 \\
$\begin{array}{l}\text { Median age, median (range) } \\
\text { PSA (ng/mL), median (range) }\end{array}$ & $63(45-79)$ & $63(51-73)$ & $66(51-74)$ \\
Pathological Stage, n (\%) & n.a. & n.a. & $9.05(2.66-35.50)$ \\
\hline pT2 & & & $23(40)$ \\
\hline pT3 & n.a. & n.a. & $34(60)$ \\
\hline Gleason Score, n (\%) & n.a. & $12(21)$ \\
\hline$<7$ & n.a. & n.a. & $39(68)$ \\
\hline$=7$ & n.a. & n.a. & $6(11)$ \\
\hline$>7$ & n.a. & n.a. & \\
\hline \hline
\end{tabular}

NPT - morphologically normal prostate tissue, PIN - prostatic intraepithelial neoplasia, PCa - prostate carcinoma, n.a. - not applicable.

\section{Overexpression of SIRT1 decreases levels of H2A.Z independently of mTOR inhibition}

To investigate the role of sirtuin 1 in the modulation of H2A.Z expression, SIRT1 overexpression was induced in LNCaP, DU145 and PC-3 cell lines [validation of successful transduction was assessed by qRT-PCR (Supplementary Fig. 1A) and Western blot (Fig. 2 A1 and 2 A2)]. Following induction of SIRT1 expression, a significant reduction of phosphorylated ribosomal protein S6 (phosphoS6, an effector of mTOR pathway) was found, although mTOR and ribosomal protein S6 (S6) remained unchanged (Figs. $2 \mathrm{~A} 1$ and $2 \mathrm{~A} 3$ ). In addition, H2A.Z protein suffered an impressive reduction to nearly undetectable levels (Fig. 2 A1), in parallel with a significant reduction of its target c-Myc, in DU145 and PC-3 cells (Fig. 2 A4).

In order to assess whether decreased H2A.Z levels were due to mTOR pathway inhibition by sirtuin 1 , the
A1
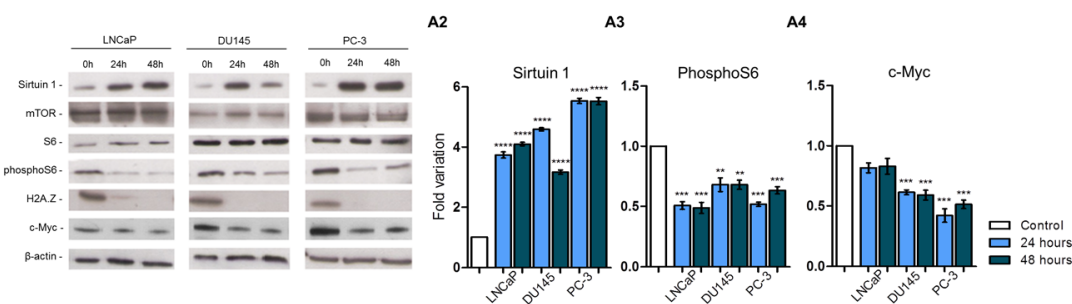

B1

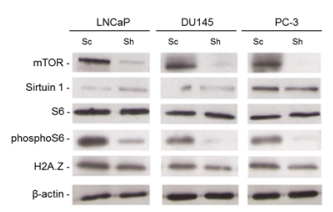

C1

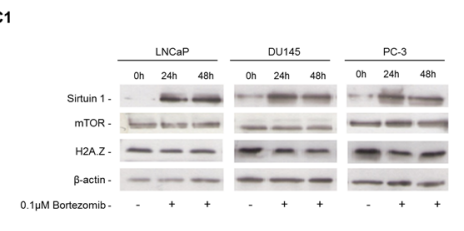

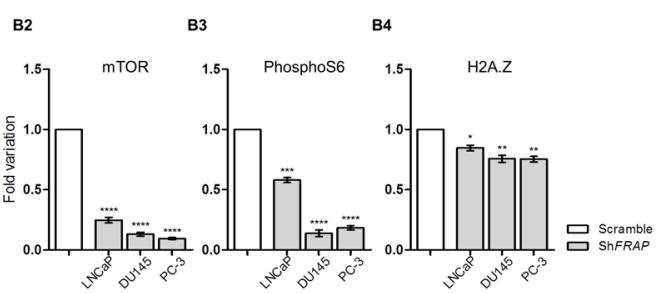

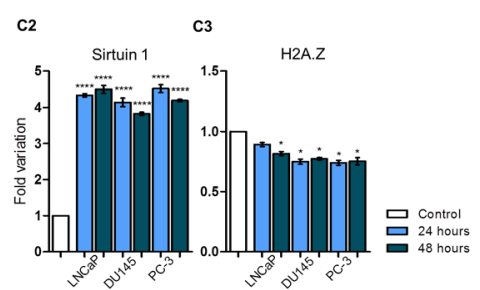

Figure 2: Protein profile of three PCa cell lines - LNCaP, DU145 and PC-3 - after (A1) SIRT1 overexpression, (B1) mTOR silencing and (C1) SIRT1 overexpression and exposure to bortezomib. After SIRT1 overexpression levels of (A2) sirtuin 1 increased, with concomitant decrease in (A3) pS6, (A4) c-Myc and H2A.Z. FRAP silencing induced a decrease in (B2) mTOR, (B3) pS6 and (B4) H2A.Z. When SIRT1 overexpressing cells were exposed to bortezomib, the increased levels of (C2) sirtuin 1 caused a significant decrease in H2A.Z levels (C3). However, this decrease was much less impressive than previously observed for SIRT1 upregulation without proteasome inhibition. Results were normalized to $\beta$-actin and are presented as fold variation in comparison to control (mean $\pm \mathrm{SD}, \mathrm{n}=3$ ). Dunnet's test: ${ }^{*} p<0.05,{ }^{* *} p<0.01,{ }^{* * *} p<0.001,{ }^{* * * *} p<0.0001$. 
selected PCa cell lines were stably silenced for FRAP, the gene encoding mTOR protein, and silencing validation was performed by qRT-PCR (Supplementary Fig. 1B) and Western blot (Fig. 2 B1 and 2 B2). As expected, mTOR pathway inhibition decreased phosphoS6 levels (Fig. 2 B3). Furthermore, a statistically significant, but modest reduction in $\mathrm{H} 2 \mathrm{~A} . \mathrm{Z}$ levels was observed following FRAP silencing (Fig. 2 B4).

\section{Pharmacological inhibition of proteasome impairs H2A.Z degradation caused by sirtuin 1 induced overexpression}

Because sirtuin 1 might induce H2A.Z degradation through the proteasome, we evaluated whether H2A.Z decrease might be due to that mechanism. Hence, the three PCa cell lines were transiently transfected for SIRT1 and exposed to $0.1 \mu \mathrm{M}$ bortezomib, a selective inhibitor of proteasomal activity (Fig. 2 C1). Although SIRT1 overexpression (Supplementary Fig. 1C and Fig. 2 C2) and concomitant proteasome inhibition were associated with a decrease in H2A.Z levels (Fig. 2 C3), the extent of this decrease was considerably less impressive when compared to SIRT1 overexpression without proteasome inhibition.

\section{Trichostatin A (TSA) leads to increased expression of both H2AFZ and SIRT1 through regulation of histone marks}

To ascertain whether epigenetic modulating drugs were able to regulate $H 2 A F Z$ and SIRT1 transcriptional
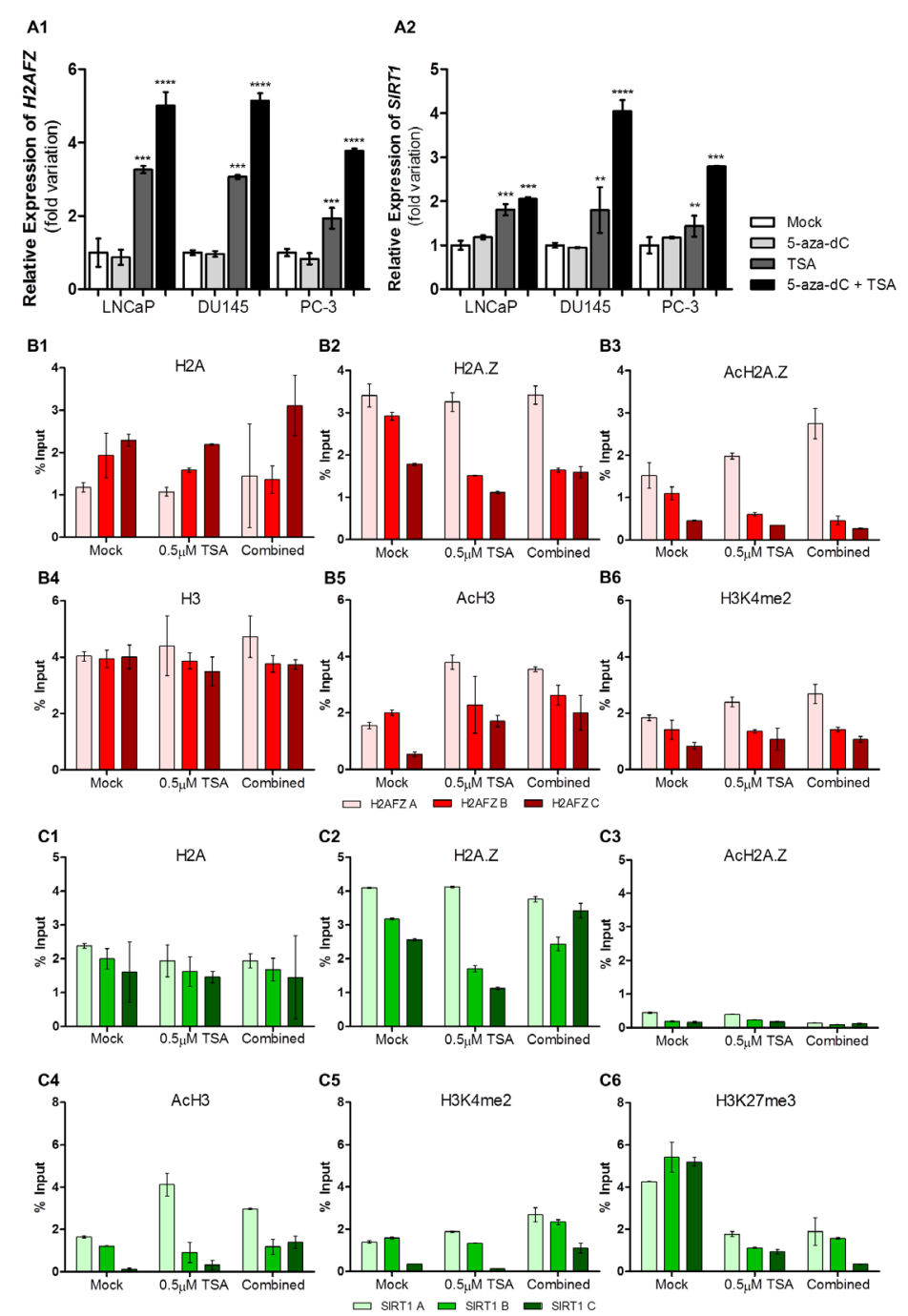

Figure 3: Epigenetic modulating drugs positively regulate $\boldsymbol{H} 2 \boldsymbol{A F Z}$ and $\boldsymbol{S I R T 1}$ transcription. Transcript levels of (A1) $H 2 A F Z$ and (A2) SIRT1 in three distinct PCa cell lines after exposure to 5-aza-dC and/or TSA. The results are presented as fold variation in comparison to the experimental control (mean $\pm \mathrm{SD}, \mathrm{n}=3$ ). Dunnet's test: $* * p<0.01, * * * p<0.001, * * * * p<0.0001$. ChIP assay results in LNCaP cell line regarding (B1) H2A, (B2) H2A.Z, (B3) AcH2A.Z, (B4) H3, (B5) AcH3 and (B6) H3K4me2 histones and histones marks across H2AFZ promoter and regarding (C1) H2A, (C2) H2A.Z, (C3) AcH2A.Z, (C4) AcH3, (C5) H3K4me2 and (C6) histones and histones marks along SIRT1 promoter. Results are normalized with the input of total sonicated chromatin (mean $\pm \mathrm{SD}$ ). 
status, PCa cell lines were exposed to 5-aza-2'depxycytidine (5-aza-dC) and/or TSA. TSA, alone or combined with 5-aza-dC, led to an increase in mRNA levels for both genes, whereas 5-aza-dC alone was not able to significantly alter their expression (Fig. 3 A1 and 3 A2). Hence, the effect of 5-aza-dC alone was not further explored.

Subsequently, chromatin immunoprecipitation (ChIP) was performed in the three selected PCa cell lines after exposure to TSA (Fig. 3B/3C and Supplementary Figs. 2, 3 and 4). For LNCaP cells, concerning $H 2 A F Z$, no relevant differences were found for $\mathrm{H} 2 \mathrm{~A}$ and $\mathrm{H} 2 \mathrm{~A} . \mathrm{Z}$ levels within the promoter, after treatment. However, differences in distribution were depicted as H2A tended to accumulate away from the TSS (Fig. $3 \mathrm{~B} 1$ ), whereas H2A.Z was enriched nearby the TSS (Fig. 3 B2), particularly its acetylated form, and after exposure to TSA (Fig. 3 B3). Concurrently, after drug exposure, H3 levels remained stable (Fig. 3 B4) whereas the distribution of the activating marks acH3 and $\mathrm{H} 3 \mathrm{~K} 4 \mathrm{me} 2$ was increased closer to the TSS (Fig. 3 B5 and 3 B6). Nevertheless, no variations were observed for the repressive mark $\mathrm{H} 3 \mathrm{~K} 27 \mathrm{me} 3$ along the full extent of the $H 2 A F Z$ promoter (Supplementary Fig. 2). Remarkably, the same results were observed for DU145 and PC-3, except for AcH2A.Z in PC-3, which was not increased after treatment with both drugs (Supplementary Fig. 3 and 4).

Likewise, in LNCaP, although H2A.Z levels were higher than those of $\mathrm{H} 2 \mathrm{~A}$, drug exposure did not alter H2A and H2A.Z levels across SIRT1 promoter (Fig. 3 $\mathrm{C} 1$ and $3 \mathrm{C} 2$ ), and both were particularly enriched nearby the TSS. Nonetheless, acH2A.Z levels remained very low and dispersed along the SIRT1 promoter (Fig. 3 C3). Within this same promoter, alterations in $\mathrm{H} 3$ levels were found in LNCaP (Supplementary Fig. 2). Additionally, an increase in histone activating marks was demonstrated in nucleosomes close to TSS (Fig. 3 C4 and 3 C5) whereas a decrease in the $\mathrm{H} 3 \mathrm{~K} 27 \mathrm{me} 3$ repressive mark was found after epigenetic drugs exposure (Fig. 3 C6). Concurrently, the same observations were found in DU145 and PC-3, although levels of $\mathrm{H} 3$ remained the same for both cell lines (Supplementary Figs. 3 and 4).

\section{Epigenetic modulating drugs reduce protein levels of $\mathrm{H} 2 \mathrm{~A} . \mathrm{Z}$ with concomitant increase of sirtuin 1}

Because SIRT1 and $H 2 A F Z$ transcript levels increased after exposure to TSA alone or combined with 5-aza-dC, H2AFZ and SIRT1 protein expression was further assessed (Fig. 4A). Intriguingly, whereas sirtuin 1 levels significantly increased after TSA exposure, alone or in combination with 5-aza-dC (Fig. 4 B1), the opposite trend was observed for H2AZ levels (Fig. 4 B2), as well as for c-Myc levels (Fig. 4 B3).

\section{Pharmacological inhibition or activation of sirtuin 1 directly affects regulation of $\mathrm{H} 2 \mathrm{~A} . \mathrm{Z}$ protein levels}

The effect of sirtuin 1 pharmacological inhibition or activation on $\mathrm{H} 2 \mathrm{~A} . \mathrm{Z}$ protein levels was assessed through exposure of PCa cell lines to the modulators of sirtuin 1 enzymatic activity nicotinamide (inhibitor) or resveratrol (activator). The same experiment was performed in combination with exposure to epigenetic modulating drugs (Figure 5).

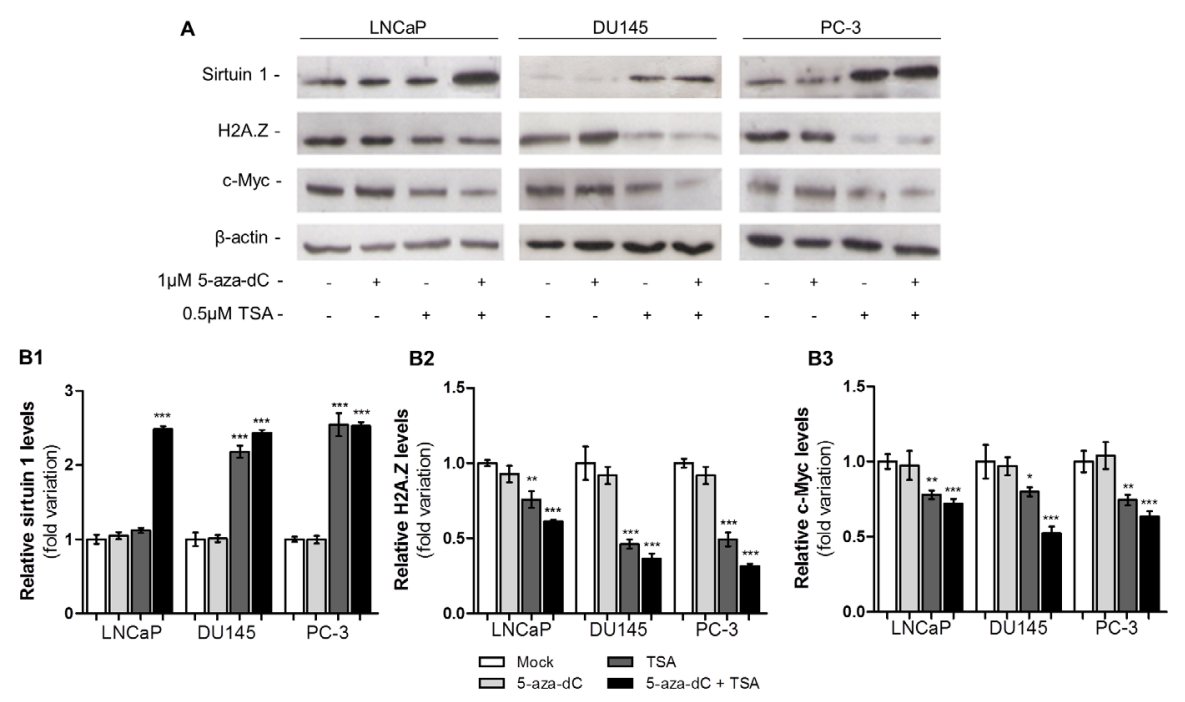

Figure 4: Epigenetic modulating drugs have the capacity to upregulate protein levels of sirtuin 1 and decrease levels of H2A.Z and c-Myc. (A) Western blot analysis of sirtuin 1, H2A.Z and c-Myc of three PCa cell lines exposed to 5-aza-dC and/or TSA. Optical densities of visible bands for (B1) sirtuin 1, (B2) H2A.Z and (B3) c-Myc following treatment with 5-aza-dC and/or TSA. Intensity of the bands was normalized to the loading control $\beta$-actin and presented as folding variation in comparison to the untreated control (mean $\pm \mathrm{SD}, \mathrm{n}=3$ ). Dunnet's test: ${ }^{*} p<0.05, * * p<0.01, * * * p<0.001$. 
Exposure to nicotinamide alone was associated with a decrease in sirtuin 1 . Whereas in LNCaP cells, sirtuin 1 levels decreased after exposure to nicotinamide combined with both epigenetic drugs, in DU145 cells the same was observed only for nicotinamide combined with TSA. On the contrary, in PC-3 cells exposed to nicotinamide combined with TSA or 5 -aza-dC plus TSA, an increase in sirtuin 1 levels was apparent (Fig. 5 A1 and 5 A2). An increase in H2A.Z protein levels after exposure to nicotinamide alone was observed only in DU145. Nonetheless, an increase in H2A.Z protein levels was observed in all cell lines when nicotinamide was combined with TSA or with both epigenetic drugs (Fig. $5 \mathrm{~A} 1$ and 5 A3).

In $\mathrm{PCa}$ cell lines exposed to resveratrol, a statistically significantly increase in sirtuin 1 protein levels was verified. This trend was more striking when the sirtuin 1 activator was used in combination with 5-aza-dC and TSA (Fig. 5 B1 and 5 B2). The opposite effect was observed for H2A.Z levels: cell lines exposed to resveratrol showed a decrease in H2A.Z levels. Interestingly, the lowest H2A.Z levels were found in PCa cell lines treated with resveratrol in combination with
5-aza-dC and TSA (Fig. $5 \mathrm{~B} 1$ and 5 B3).

Finally, in PC-3 cell line exposed to epigenetic modulating drugs, a statistically significant increase in the interaction between sirtuin 1 and H2A.Z was demonstrated using PLA, and the same result was observed following exposure to resveratrol alone (Figs. 6A, 6B). Additionally, when cells were exposed to nicotinamide, a total absence of signals was depicted (data not shown).

\section{DISCUSSION}

Histone variants seem to play a major role in gene expression regulation. Specifically, H2A.Z and acH2A.Z have been shown to be deregulated in some tumors, causing aberrant expression of several oncogenes $[9,15$, 27, 28]. Moreover, sirtuin 1, a protein that may acts either as tumor suppressor or oncoprotein, has been previously shown to reduce H2A.Z levels in cardiomyocytes, via a cascade of events that ultimately leads to proteasomemediated histone degradation [16]. Thus, we aimed to characterize the role of H2A.Z deregulation in prostate carcinogenesis and determine how sirtuin 1 might be involved in this process. After confirmation of

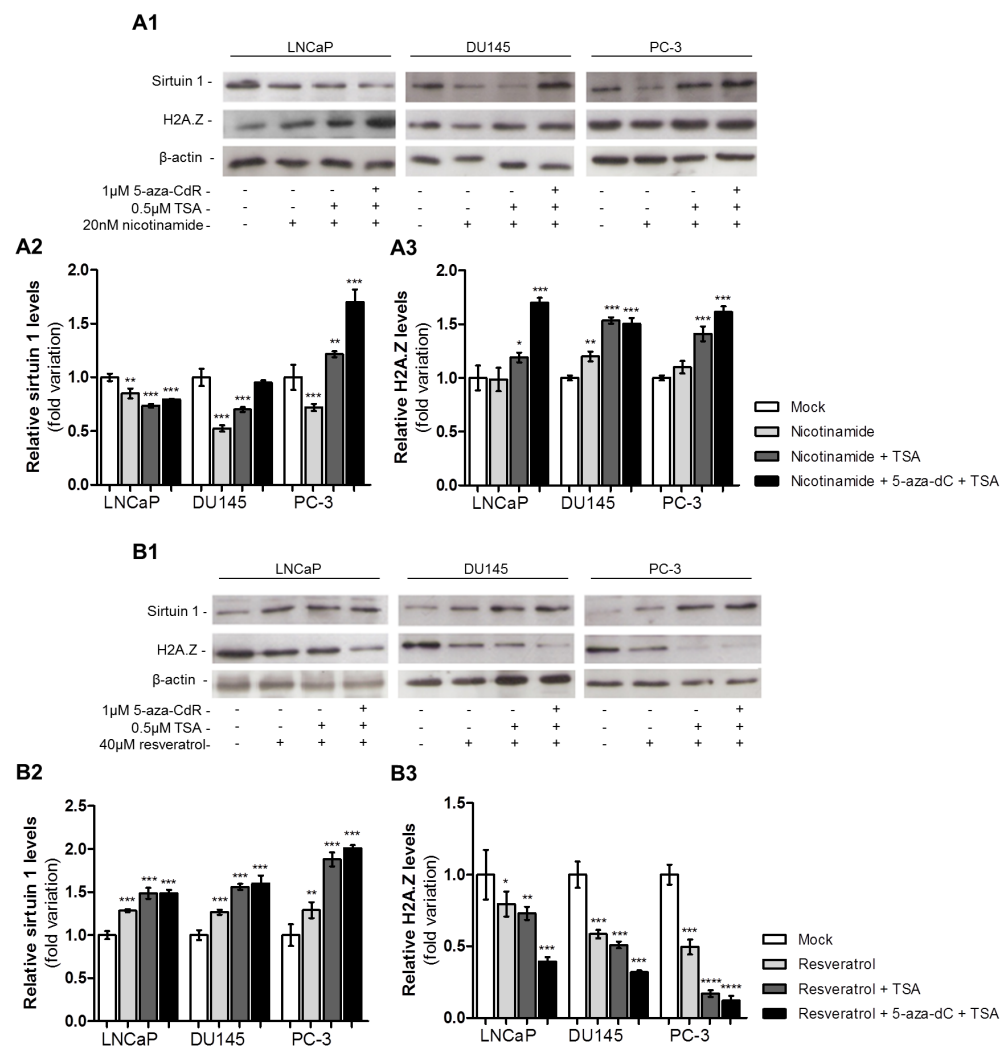

Figure 5: Pharmacological inhibition or activation of sirtuin 1 promotes H2A.Z up- or downregulation. (A1) Western blot analysis of sirtuin 1 and H2A.Z of three PCa cell lines exposed to nicotinamide, alone or combined with 5-aza-dC and/or TSA. Optical densities of visible bands for (A2) sirtuin 1 and (A3) H2A.Z following drug exposure. (B1) Western blot analysis of sirtuin 1 and H2A.Z of three $\mathrm{PCa}$ cell lines exposed to resveratrol, alone or combined with 5-aza-dC and/or TSA. Optical densities of visible bands for (B2) sirtuin 1 and (B3) H2A.Z following drug exposure. Intensity of bands was normalized to the loading control $\beta$-actin and presented as folding variation in comparison to the untreated control (mean $\pm \mathrm{SD}, \mathrm{n}=3$ ). Dunnet's test: ${ }^{*} p<0.05, * * p<0.01,{ }^{* * *} p<0.001$. 
deregulation of SIRT1 and $H 2 A F Z$ expression in primary PCa tissues, we found that, in PCa cell lines, induction of SIRT1 expression led to a dramatic decrease of H2A.Z levels, mostly through proteasome-mediated degradation. Furthermore, exposure to epigenetic modulating drugs, mainly TSA, was able to reverse the concomitant deregulation of both genes in $\mathrm{PCa}$ at transcript and protein level. In addition to histone modification, direct interaction of sirtuin 1 and H2A.Z at protein level is also involved in sirtuin 1 regulation of $H 2 A F Z$ expression.

This is the first report of altered $H 2 A F Z$ expression in localized primary $\mathrm{PCa}$, as well as in its putative precursor lesion, PIN. Interestingly, there is a previous publication on a trend for increased H2A.Z expression in tumors from patients submitted to androgen deprivation therapy, suggestive of a subset of castration-resistant tumors over-expressing this histone variant [14]. Concurrently, SIRT1 decreased expression was found in the same dataset. This observation is, however, conflicting with some previous studies [22], but in line with others [21]. Our findings sustain the downregulation of SIRT1 in $\mathrm{PCa}$, as well as in PIN lesions, supporting the hypothesis that sirtuin 1 is involved in $H 2 A F Z$ regulation. It should be recalled that SIRT1 has been considered an oncogene and a tumor suppressor, depending on the cancer model under consideration [17, 29]. Indeed, it is known that sirtuin 1 is responsible for acquisition of genetic mutations in $B C R-A B L$ in chronic myelogenous leukemia cells, conferring resistance to imatinib mesylate, which inhibits that tyrosine kinase [30, 31]. Additionally, SIRT1 inhibits p53 through deacetylation, thus increasing the possibility of neoplastic transformation [32], p53 regulates SIRT1 transcription through a negative feedback loop, decreasing SIRT1 activity, which, in turn, increases TP53 activity [33]. Moreover, SIRT1 has been suggested as a tumor suppressor in colon cancer due to its ability to deacetylate and inactivate oncogenic $\beta$ - catenin [23]. In the same vein, BRCA1 enhances sirtuin 1 activity, reducing cell growth and increasing apoptosis in breast cancer cells [25]. In PCa, sirtuin 1 has been involved in autophagy, and SIRT1 knockdown induced PIN development, suggesting that SIRT1 might be a tumor suppressor gene in this cancer model [24].

We found that SIRT1 induced overexpression in PCa cell lines decreased H2A.Z protein levels. Since sirtuin 1 inhibits the mTOR pathway [26], we speculated whether H2A.Z downregulation due to sirtuin 1 overexpression might be mediated by repression of mTOR pathway,
A
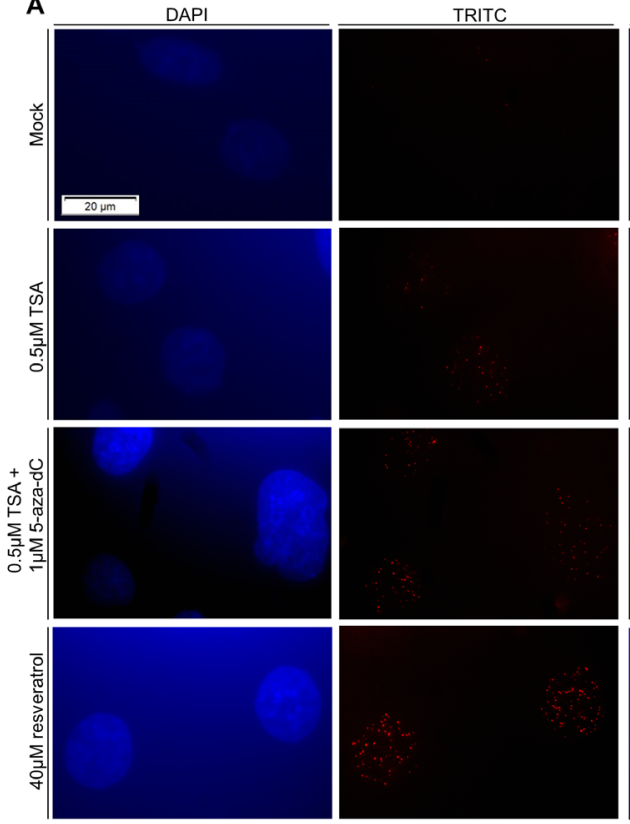

B

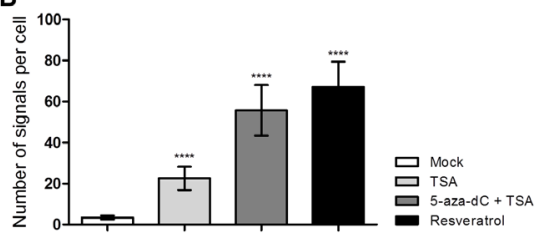

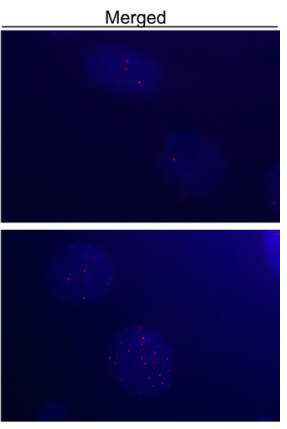
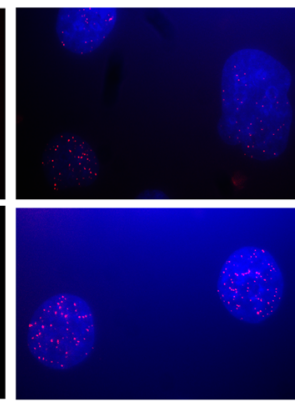

D-aza- $d C+T S A$
Resveratrol

Figure 6: PCa cells exposure to epigenetic modulating drugs and resveratrol promotes the physical and direct interaction between sirtuin 1 and H2A.Z. (A) PLA assay for PC-3 after exposure to $0.5 \mu \mathrm{M}$ TSA, alone or combined with $1 \mu \mathrm{M}$ 5 -aza-dC, and $40 \mu \mathrm{M}$ resveratrol (40x magnification). (B) Analysis of PLA results represented as number of signals per cell (mean \pm SD, $\mathrm{n}=50)$. Dunnet's test: $* * * * p<0.0001$. 
which is overactive and associated with tumor progression in PCa [34, 35]. Our findings suggest that, although mTOR inhibition might contribute to sirtuin 1-related downregulation of $\mathrm{H} 2 \mathrm{~A} . \mathrm{Z}$, it is not the main responsible as FRAP stably silenced PCa cell lines showed only a slight decrease in H2A.Z levels. Thus, other mechanisms are involved in regulation of $\mathrm{H} 2 \mathrm{~A} . \mathrm{Z}$ by sirtuin 1 . In fact, when simultaneous SIRT1 overexpression and exposure to bortezomib were performed, levels of H2A.Z modestly decreased, depicting that proteasome has a major importance in sirtuin 1-mediated H2A.Z degradation, as previously described [16].

Because sirtuin 1 is a Class III HDAC, we hypothesized that post-translational histone modification might be involved in $H 2 A F Z$ deregulation in PCa. Remarkably, exposure to epigenetic drugs (TSA alone or in combination with 5-aza-dC) resulted in increased $H 2 A F Z$ levels in $\mathrm{PCa}$ cells and these results were corroborated by ChIP, which demonstrated an enrichment of histone activating marks, such as $\mathrm{AcH} 3$ and $\mathrm{H} 3 \mathrm{~K} 4 \mathrm{me} 2$, in the TSS proximity. Nonetheless, H2A and H2A.Z levels remained stable after drug exposure and the deposition pattern of both histones suggest that H2A.Z, and especially acH2A.Z, replace $\mathrm{H} 2 \mathrm{~A}$ in nucleosomes next to the TSS, which makes the gene prone to be actively transcribed. Interestingly, this pattern is characteristic of oncogenes and actively transcribed genes [15, 36, 37], and supports an oncogenic function for $H 2 A F Z$. Nevertheless, and despite increased gene transcription, protein levels of H2A.Z decreased after exposure to TSA, indicating that a post-translational mechanism was involved in regulation of H2A.Z levels. Based on our results, we postulate that sirtuin 1 is responsible for that regulation. Indeed, we found that SIRT1 expression was increased, both at transcript and protein levels, in all PCa cell lines after treatment with TSA. Moreover, enrichment in activating marks - specifically acH3 and $\mathrm{H} 3 \mathrm{~K} 4 \mathrm{me} 2$ - was observed near the TSS of SIRT1 gene, with a simultaneous decrease of the repressive mark H3K27me3. Hence, our results strongly suggest that epigenetic modulating drugs are able to increase SIRT1 expression through histone post translational modifications. Remarkably, in contrast to $H 2 A F Z$, although levels of H2A.Z were increased nearby the SIRT1 TSS, the canonical histone was not depleted, depicting a common feature of poised or poorly transcribed genes in cancer cells [15]. Importantly, we demonstrated that H2A.Z is not acetylated within nucleosomes neighboring SIRT1 TSS, either before or after TSA exposure, which is in accordance with a previous study in PCa, regarding TSG [15]. Interestingly, it has been reported that sirtuin 1 not only regulates $\mathrm{H} 2 \mathrm{~A} . \mathrm{Z}$ levels, but also that H2A.Z itself might influence SIRT1 expression, suggesting a reciprocal regulation mechanism between both proteins $[7,16]$. Thus, we are tempted to speculate that H2A.Z overexpression negatively regulates SIRT1, resulting in the lower sirtuin 1 levels observed in PCa cell lines and primary tissues.

To ascertain the impact of sirtuin 1 activity on H2A.Z levels, we tested an inhibitor (nicotinamide) and an activator (resveratrol) of sirtuin 1 alone or combined with epigenetic modulating drugs. Remarkably, the effect on H2A.Z protein levels was independent of exposure to the epigenetic modifying drugs, demonstrating that sirtuin 1 also regulates H2A.Z levels through its direct enzymatic activity. Intriguingly, we found that nicotinamide induced a decrease of sirtuin 1 levels and similar results have already been reported in leukemic cells [38]. Contrarily, resveratrol alone induced an increase in sirtuin 1 protein levels, which is line with previous reports showing that it promotes SIRT1 expression [39]. Hence, the combined
A
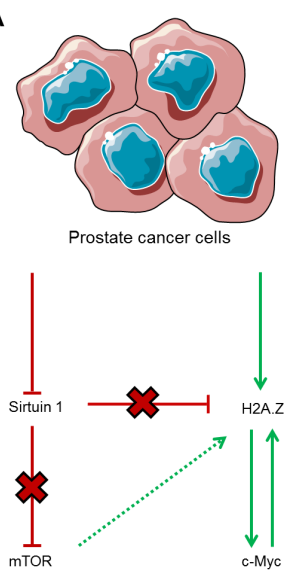

B

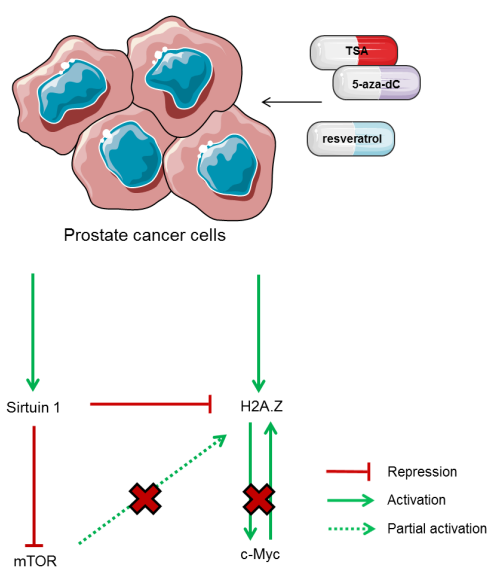

Figure 7: Interplay among sirtuin 1, H2A.Z, mTOR and c-Myc in prostate cancer cells. (A) Under normal conditions, PCa cells display downregulation of SIRT1, thus, sirtuin 1 is unable to promote H2A.Z negative regulation directly, through its degradation, and indirectly, through mTOR pathway repression, leading to upregulation of c-Myc and other oncogenes. (B) When exposed to TSA, alone or combined with 5-aza-dC, or resveratrol, levels of sirtuin 1 are restored, leading to decreased levels of H2A.Z, partially due to mTOR pathway inhibition, but mostly via direct interaction between sirtuin 1 and the histone variant. Similarly, c-Myc levels decrease after treatment with those drugs. 
effect of activation and induced expression of sirtuin 1 by resveratrol substantiates the observed decrease in H2A.Z protein levels. Importantly, exposure to epigenetic modifying drugs augmented this effect, mainly through increased SIRT1 expression, as expected.

Importantly, we found that the mechanism by which sirtuin 1 promotes H2A.Z downregulation involves proteasomal-mediated degradation. This mechanism has been previously demonstrated in cardiomyocytes, in which increased deacetylation of Lys-15 triggers H2A.Z ubiquitylation on Lys-115 and Lys-121 and, ultimately, its degradation by the proteasomal pathway [16]. To further support this hypothesis in PCa cells, we showed, through PLA, that exposure to epigenetic modulating drugs dramatically increased the interaction between sirtuin 1 and $\mathrm{H} 2 \mathrm{~A} . \mathrm{Z}$, promoting their physical contact which is critical for degradation.

Interestingly, H2A.Z, a histone variant consistently associated with the malignant phenotype, might constitute an interesting therapeutic target [27]. Concerning the mechanism that regulates $\mathrm{H} 2 \mathrm{~A} . \mathrm{Z}$ incorporation into nucleosomes, the ATP dependent nucleosome remodeler SNF-2-related CREB-binding protein activator protein (SRCAP) inserts H2A.Z into nucleosomes and deposits it near gene promoters [40, 41]. Remarkably, SRCAP has been already reported to be overexpressed in $\mathrm{PCa}$ [42]. Additionally, p400/Tip60, another complex responsible for H2A.Z insertion into chromatin, is also overactive in PCa [43]. Although a therapy based on SRCAP or p400/Tip60 repression would seem attractive, specific targeting of those complexes might entail high toxicity to non-neoplastic cells. Furthermore, it has been reported that H2A.Z and c-Myc, a transcription factor highly expressed in $\mathrm{PCa}$ [44], positively regulate each other, with high levels of the histone variant colocalized in $C-M Y C$ promoter during active transcription [9, 12]. Hence, targeting of c-Myc might not only regulate this protein, but it would also allow for indirect regulation of H2A.Z. Nevertheless, anti-c-Myc based therapy is changeling and displays high toxicity, since $C-M Y C$ expression is ubiquitous in all proliferating, including non-malignant, cells [45].

Interestingly, a close correlation between sirtuin 1 and c-Myc has been already reported, and a synergistic effect has been demonstrate for these two proteins [4649], suggesting an oncogenic role for sirtuin 1. However, it is known that the oncogenic role of sirtuin 1 is tumorand context-specific, and the opposite relation between the histone deacetylase and c-Myc has already been described, indicating that sirtuin 1 promotes c-Myc instability [50]. Although in a different mechanistic context, we also observed a negative correlation between sirtuin 1 and c-Myc oncoprotein. Hence, we demonstrated that epigenetic modulation of $\mathrm{H} 2 \mathrm{~A} . \mathrm{Z}$, mediated by sirtuin 1 upregulation, decreased c-Myc expression, which might provide a more effective and less toxic alternative.
In conclusion, we showed that sirtuin 1 and H2A.Z deregulation in PCa are reciprocally related. Epigenetic mechanisms, mostly histone post-translational modifications, are likely involved and impair sirtuin 1-mediated downregulation of H2A.Z via proteasomemediated degradation. Epigenetic modifying drugs in conjunction with enzymatic modulators are able to restore the normal functions of sirtuin 1 and might constitute relevant tools for targeted therapy of PCa patients (Fig. 7).

\section{MATERIALS AND METHODS}

\section{Patients and Samples}

Fifty-seven PCa samples were prospectively collected from patients diagnosed and treated with radical prostatectomy at the Portuguese Oncology Institute - Porto, Portugal. From 10 of those patients, samples of PIN were also collected. Ten NPT, used as controls, were obtained from the peripheral zone of prostates that did not harbor PCa of patients submitted to radical cystoprostatectomy for bladder cancer. After surgery, all tissues specimens were promptly frozen at $-80^{\circ} \mathrm{C}$ and subsequently cut in a cryostat for nucleic acid extraction.

From each specimen, fragments were routinely collected, formalin-fixed and paraffin-embedded for histopathological examination. An expert pathologist evaluated Gleason score and pathological staging. Relevant clinical data was collected from the clinical charts. This study was approved by the institutional review board (Comissão de Ética para a Saúde) of Portuguese Oncology Institute - Porto, Portugal.

\section{Prostate cancer cells}

LNCaP cells were grown in RPMI 1640, DU145 cells were maintained in MEM and PC-3 cells were grown in 50\% RPMI-50\% F-12 medium (GIBCO), as recommended. All culture media were supplemented with $10 \%$ fetal bovine serum and $1 \%$ penicillin/streptomycin (GIBCO). Cells were maintained in an incubator at $37^{\circ} \mathrm{C}$ with $5 \% \mathrm{CO}_{2}$. All PCa cell lines were karyotyped by G-banding (for validation purposes) and routinely tested for Mycoplasma spp. contamination (PCR Mycoplasma Detection Set, Clontech Laboratories).

\section{Transient transfection}

PCa cells were transiently transfected with transfection-ready DNA containing SIRT1 transcript variant 1 (Origene Technologies), following manufacturer's instructions. After transfection, SIRT1overexpressing cells were also exposed to $0.1 \mu \mathrm{M}$ 
bortezomib (Cell Signaling) and subsequently harvested for RNA and protein extraction.

\section{Short hairpin RNA (shRNA) and lentiviral transduction}

Cells were transfected with mTOR or negative control shRNA using shRNA Lentiviral Particles (sc35409-V and sc-108080, respectively, Santa Cruz Biotechnology), according to manufacturer's indications. Clones successfully silenced were selected using the recommended complete medium supplemented with 2-5 $\mu \mathrm{g} / \mathrm{mL}$ puromycin, as recommended by manufacturer. Cells were then collected for RNA and protein extraction.

\section{Cell culture and treatment with epigenetic modulating drugs, nicotinamide and resveratrol}

PCa cells treated with $1 \mu \mathrm{M} 5$-aza-dC (Sigma) for $72 \mathrm{~h}$ and $0.5 \mu \mathrm{M}$ TSA (Sigma), added the last $24 \mathrm{~h}$. Cells were also exposed to $20 \mathrm{mM}$ nicotinamide (Sigma) or $40 \mu \mathrm{M}$ resveratrol (Sigma) for $72 \mathrm{~h}$, both alone or in combination with TSA or 5-aza-dC and TSA, according to the previous regimen. For control purposes, the same cell lines were cultured without treatment for 72 hours (mock cells). For each condition, three biological replicates were performed. Both medium and drugs were replaced every $24 \mathrm{~h}$.

\section{RNA isolation and quantitative reverse- transcription PCR (qRT-PCR)}

Total RNA from tissue samples and cell lines was isolated using Trizol ${ }^{\circledR}$ Reagent (Invitrogen) and all genomic DNA present in the samples was eliminated using TURBO DNA-free (Ambion, Applied Biosystems), following manufacturer's instructions. For each sample, first strand synthesis was performed using the HighCapacity cDNA Reverse Transcription Kit (Applied Biosystems). Expression of target genes was quantified using TaqMan expression assays [H2AFZ (Hs01888362 g1), SIRT1 (Hs01009005_m1), FRAP (Hs00234508_ $\mathrm{m} 1)$ ], acquired as pre-developed assays from Applied Biosystems and normalized to the expression of the endogenous control GUSB (Hs99999908 m1). The standard curve method was used to determine RNA levels. All samples were analysed in triplicate, and the mean value was used for data analysis.

\section{Whole-cell extracts and immunoblot}

Whole-cell protein extraction was performed using complete RIPA Buffer (Santa Cruz Biotechnology Inc.).
Briefly, $20 \mu \mathrm{g}$ of total protein were separated by SDSPAGE, transferred to nitrocellulose membranes and probed with antibodies against H2A.Z (Cell Signaling), sirtuin 1 (Abcam), mTOR (Cell Signaling), S6 (Cell Signaling), pS6 (Cell Signaling), c-Myc (Abcam) or the endogenous control $\beta$-actin (Sigma). Finally, blots were developed using Immun-Star WesternC Kit, according to manufacturer's indications (BioRad). All the experiments were performed in triplicate. Relative optical density determination was performed using QuantityOne Software version 4.6.6. (Biorad) and normalized for the loading control, $\beta$-actin.

\section{Chromatin immunoprecipitation (ChIP) Assay}

EZ-Magna ChIP G-Chromatin Immunoprecipitation Kit and the Magna Grip Rack (Millipore) were used to perform ChIP assay according to the manufacturer's instructions. For each chromatin immunoprecipitation assay, anti-H2A (Abcam), anti-H2A.Z (Abcam), antiAcH2A.Z (Abcam), anti-H3 (Abcam), anti-AcH3 (Millipore), anti-H3K4me2 (Abcam), anti-Histone H3K27me3 (Millipore) and the negative control provided with the kit (normal mouse IgG), were used.

DNA quantification was performed in a 7500 RealTime PCR System (Applied Biosystems), using Power SYBR Green PCR Master Mix (Applied Biosystems). Three gene-specific pairs of primers for each gene promoter were used, in which primers A were located closer to TSS and C those that were more distant upstream TSS (Supp. Table 1). The relative amount of promoter DNA was normalized using Input Percent Method.

\section{Proximity ligation assay (PLA)}

PC-3 cells, cultured in $1 \mathrm{~cm}^{2}$ coverslips, were exposed to $0.5 \mu \mathrm{M}$ TSA (Sigma), alone or combined with $1 \mu \mathrm{M} 5$-aza-dC (Sigma), and to $40 \mu \mathrm{M}$ resveratrol (Sigma), as previously mentioned. Additionally, Mock cells were used as controls. At the end of treatment, cells were fixed in $4 \%$ formaldehyde (Sigma) for 10 minutes and permeabilized in $0.5 \%$ Triton X-100 (Sigma), for 5 minutes, at room temperature and gently stirred.

PLA assay was performed using the commercial kit Duolink In Situ (OLINK Bioscience), according to manufacturer's instructions. The antibodies used were anti-sirtuin 1 (Abcam) and anti-H2A.Z (Cell Signaling). After the procedure, slides were evaluated under a fluorescence microscope (Olympus IX51, Olympus). PLA signals were counted in fifty randomly selected cells for each treatment. 


\section{Statistical analysis}

In cell lines, differences in transcript and protein levels among the treatments performed were determined using One-Way Analysis of Variance (one-Way ANOVA), followed by a multiple comparison Dunnet's (post-hoc) test, or Student's t-test, as appropriate, comparing all groups against the Mock.

Differences in quantitative expression levels of SIRT1 and H2AFZ among NPT, PIN and PCA were assessed by the nonparametric Kruskall-Wallis test, followed by pairwise comparisons through Mann-Whitney $U$-test with Bonferroni's correction, when appropriate. The relationship between expression levels and standard clinicopathological variables (Gleason score, pathological stage) were assessed using the Kruskall- Wallis or the Mann-Whitney tests, as appropriate.

All tests were two-sided and statistical significance was set at $p<0.05$. Statistical analysis was performed using GraphPad Prism version 5.0 for Windows (GraphPad Software).

\section{ACKNOWLEDGMENTS}

This study was supported by grants from CIIPOP-4-2008 Research Center of Portuguese Oncology Institute Porto and Fundação para a Ciência e Tecnologia (FCT; PEST-OE/SAL/UI0776/2011). Additional funds were provided by a grant from the European Community's Seventh Framework Programme - Grant number FP7HEALTH-F5-2009-241783. IG was supported by grants from FCT - Fundação para a Ciência e a Tecnologia [SFRH/BD/64082/2009], ES and PCP were supported by a grant from Liga Portuguesa Contra o Cancro - Núcleo Regional do Norte and AIO and NRC were research fellows from FP7-HEALTH-F5-2009-241783.

\section{CONFLICT OF INTERESTS}

None of the authors have any conflict of interest to declare.

\section{REFERENCES}

1. Siegel R, Naishadham D and Jemal A. Cancer statistics, 2012. CA: a cancer journal for clinicians. 2012; 62(1):1029.

2. Shen MM and Abate-Shen C. Molecular genetics of prostate cancer: new prospects for old challenges. Genes \& development. 2010; 24(18):1967-2000.

3. Jeronimo C, Bastian PJ, Bjartell A, Carbone GM, Catto JW, Clark SJ, Henrique R, Nelson WG and Shariat SF. Epigenetics in prostate cancer: biologic and clinical relevance. European urology. 2011; 60(4):753-766.
4. Altaf M, Auger A, Covic M and Cote J. Connection between histone $\mathrm{H} 2 \mathrm{~A}$ variants and chromatin remodeling complexes. Biochemistry and cell biology = Biochimie et biologie cellulaire. 2009; 87(1):35-50.

5. Rangasamy D, Greaves I and Tremethick DJ. RNA interference demonstrates a novel role for H2A.Z in chromosome segregation. Nature structural \& molecular biology. 2004; 11(7):650-655.

6. Dhillon N, Oki M, Szyjka SJ, Aparicio OM and Kamakaka RT. H2A.Z functions to regulate progression through the cell cycle. Molecular and cellular biology. 2006; 26(2):489501.

7. Meneghini $\mathrm{MD}, \mathrm{Wu} \mathrm{M}$ and Madhani HD. Conserved histone variant H2A.Z protects euchromatin from the ectopic spread of silent heterochromatin. Cell. 2003; 112(5):725-736.

8. Dunican DS, McWilliam P, Tighe O, Parle-McDermott A and Croke DT. Gene expression differences between the microsatellite instability (MIN) and chromosomal instability (CIN) phenotypes in colorectal cancer revealed by high-density cDNA array hybridization. Oncogene. 2002; 21(20):3253-3257.

9. Hua S, Kallen CB, Dhar R, Baquero MT, Mason CE, Russell BA, Shah PK, Liu J, Khramtsov A, Tretiakova MS, Krausz TN, Olopade OI, Rimm DL and White KP. Genomic analysis of estrogen cascade reveals histone variant H2A.Z associated with breast cancer progression. Molecular systems biology. 2008; 4:188.

10. Zucchi I, Mento E, Kuznetsov VA, Scotti M, Valsecchi V, Simionati B, Vicinanza E, Valle G, Pilotti S, Reinbold R, Vezzoni P, Albertini A and Dulbecco R. Gene expression profiles of epithelial cells microscopically isolated from a breast-invasive ductal carcinoma and a nodal metastasis. Proceedings of the National Academy of Sciences of the United States of America. 2004; 101(52):18147-18152.

11. Rhodes DR, Yu J, Shanker K, Deshpande N, Varambally R, Ghosh D, Barrette T, Pandey A and Chinnaiyan AM. Large-scale meta-analysis of cancer microarray data identifies common transcriptional profiles of neoplastic transformation and progression. Proceedings of the National Academy of Sciences of the United States of America. 2004; 101(25):9309-9314.

12. Farris SD, Rubio ED, Moon JJ, Gombert WM, Nelson $\mathrm{BH}$ and Krumm A. Transcription-induced chromatin remodeling at the c-myc gene involves the local exchange of histone H2A.Z. The Journal of biological chemistry. 2005; 280(26):25298-25303.

13. Marques M, Laflamme L, Gervais AL and Gaudreau L. Reconciling the positive and negative roles of histone H2A.Z in gene transcription. Epigenetics : official journal of the DNA Methylation Society. 2010; 5(4):267-272.

14. Dryhurst D, McMullen B, Fazli L, Rennie PS and Ausio J. Histone H2A.Z prepares the prostate specific antigen (PSA) gene for androgen receptor-mediated transcription and is upregulated in a model of prostate cancer progression. 
Cancer letters. 2012; 315(1):38-47.

15. Valdes-Mora F, Song JZ, Statham AL, Strbenac D, Robinson MD, Nair SS, Patterson KI, Tremethick DJ, Stirzaker C and Clark SJ. Acetylation of H2A.Z is a key epigenetic modification associated with gene deregulation and epigenetic remodeling in cancer. Genome research. 2012; 22(2):307-321.

16. Chen IY, Lypowy J, Pain J, Sayed D, Grinberg S, Alcendor RR, Sadoshima J and Abdellatif M. Histone H2A.z is essential for cardiac myocyte hypertrophy but opposed by silent information regulator 2alpha. The Journal of biological chemistry. 2006; 281(28):19369-19377.

17. Bosch-Presegue $\mathrm{L}$ and Vaquero A. The dual role of sirtuins in cancer. Genes \& cancer. 2011; 2(6):648-662.

18. Deng CX. SIRT1, is it a tumor promoter or tumor suppressor? International journal of biological sciences. 2009; 5(2):147-152.

19. Bradbury CA, Khanim FL, Hayden R, Bunce CM, White DA, Drayson MT, Craddock C and Turner BM. Histone deacetylases in acute myeloid leukaemia show a distinctive pattern of expression that changes selectively in response to deacetylase inhibitors. Leukemia. 2005; 19(10):1751-1759.

20. Stunkel W, Peh BK, Tan YC, Nayagam VM, Wang X, Salto-Tellez M, Ni B, Entzeroth M and Wood J. Function of the SIRT1 protein deacetylase in cancer. Biotechnology journal. 2007; 2(11):1360-1368.

21. Wang RH, Sengupta K, Li C, Kim HS, Cao L, Xiao C, Kim $\mathrm{S}$, Xu X, Zheng Y, Chilton B, Jia R, Zheng ZM, Appella E, Wang XW, Ried T and Deng CX. Impaired DNA damage response, genome instability, and tumorigenesis in SIRT1 mutant mice. Cancer cell. 2008; 14(4):312-323.

22. Huffman DM, Grizzle WE, Bamman MM, Kim JS, Eltoum IA, Elgavish A and Nagy TR. SIRT1 is significantly elevated in mouse and human prostate cancer. Cancer research. 2007; 67(14):6612-6618.

23. Firestein R, Blander G, Michan S, Oberdoerffer P, Ogino $\mathrm{S}$, Campbell J, Bhimavarapu A, Luikenhuis S, de Cabo R, Fuchs C, Hahn WC, Guarente LP and Sinclair DA. The SIRT1 deacetylase suppresses intestinal tumorigenesis and colon cancer growth. PloS one. 2008; 3(4):e2020.

24. Powell MJ, Casimiro MC, Cordon-Cardo C, He X, Yeow WS, Wang C, McCue PA, McBurney MW and Pestell RG. Disruption of a Sirt1-dependent autophagy checkpoint in the prostate results in prostatic intraepithelial neoplasia lesion formation. Cancer research. 2011; 71(3):964-975.

25. Wang RH, Zheng Y, Kim HS, Xu X, Cao L, Luhasen T, Lee MH, Xiao C, Vassilopoulos A, Chen W, Gardner K, Man YG, Hung MC, Finkel T and Deng CX. Interplay among BRCA1, SIRT1, and Survivin during BRCA1-associated tumorigenesis. Molecular cell. 2008; 32(1):11-20.

26. Ghosh HS, McBurney $M$ and Robbins PD. SIRT1 negatively regulates the mammalian target of rapamycin. PloS one. 2010; 5(2):e9199.

27. Rangasamy D. Histone variant H2A.Z can serve as a new target for breast cancer therapy. Current medicinal chemistry. 2010; 17(28):3155-3161.

28. Svotelis A, Gevry N, Grondin G and Gaudreau L. H2A.Z overexpression promotes cellular proliferation of breast cancer cells. Cell Cycle. 2010; 9(2):364-370.

29. Fusco S, Maulucci G and Pani G. Sirt1: def-eating senescence? Cell Cycle. 2012; 11(22):4135-4146.

30. Chen W. Accelerating cancer evolution: a dark side of SIRT1 in genome maintenance. Oncotarget. 2012; 3(4):363364.

31. Wang Z, Yuan H, Roth M, Stark JM, Bhatia R and Chen WY. SIRT1 deacetylase promotes acquisition of genetic mutations for drug resistance in CML cells. Oncogene. 2013; 32(5):589-598.

32. Brooks $\mathrm{CL}$ and $\mathrm{Gu}$ W. p53 Activation: a case against Sir. Cancer cell. 2008; 13(5):377-378.

33. Nemoto S, Fergusson MM and Finkel T. Nutrient availability regulates SIRT1 through a forkhead-dependent pathway. Science. 2004; 306(5704):2105-2108.

34. Kinkade CW, Castillo-Martin M, Puzio-Kuter A, Yan J, Foster TH, Gao H, Sun Y, Ouyang X, Gerald WL, CordonCardo $\mathrm{C}$ and Abate-Shen C. Targeting AKT/mTOR and ERK MAPK signaling inhibits hormone-refractory prostate cancer in a preclinical mouse model. The Journal of clinical investigation. 2008; 118(9):3051-3064.

35. Kremer CL, Klein RR, Mendelson J, Browne W, Samadzedeh LK, Vanpatten K, Highstrom L, Pestano GA and Nagle RB. Expression of mTOR signaling pathway markers in prostate cancer progression. The Prostate. 2006; 66(11):1203-1212.

36. Halley JE, Kaplan T, Wang AY, Kobor MS and Rine J. Roles for H2A.Z and its acetylation in GAL1 transcription and gene induction, but not GAL1-transcriptional memory. PLoS biology. 2010; 8(6):e1000401.

37. Bruce K, Myers FA, Mantouvalou E, Lefevre P, Greaves I, Bonifer C, Tremethick DJ, Thorne AW and Crane-Robinson C. The replacement histone H2A.Z in a hyperacetylated form is a feature of active genes in the chicken. Nucleic acids research. 2005; 33(17):5633-5639.

38. Audrito V, Vaisitti T, Rossi D, Gottardi D, D'Arena G, Laurenti L, Gaidano G, Malavasi F and Deaglio S. Nicotinamide blocks proliferation and induces apoptosis of chronic lymphocytic leukemia cells through activation of the p53/miR-34a/SIRT1 tumor suppressor network. Cancer research. 2011; 71(13):4473-4483.

39. Nakayama H, Yaguchi T, Yoshiya S and Nishizaki T. Resveratrol induces apoptosis MH7A human rheumatoid arthritis synovial cells in a sirtuin 1-dependent manner. Rheumatology international. 2012; 32(1):151-157.

40. Wong MM, Cox LK and Chrivia JC. The chromatin remodeling protein, SRCAP, is critical for deposition of the histone variant H2A.Z at promoters. The Journal of biological chemistry. 2007; 282(36):26132-26139.

41. Yang X, Noushmehr H, Han H, Andreu-Vieyra C, Liang G 
and Jones PA. Gene reactivation by 5-aza-2'-deoxycytidineinduced demethylation requires SRCAP-mediated H2A.Z insertion to establish nucleosome depleted regions. PLoS genetics. 2012; 8(3):e1002604.

42. Slupianek A, Yerrum S, Safadi FF and Monroy MA. The chromatin remodeling factor SRCAP modulates expression of prostate specific antigen and cellular proliferation in prostate cancer cells. Journal of cellular physiology. 2010; 224(2):369-375.

43. Halkidou K, Gnanapragasam VJ, Mehta PB, Logan IR, Brady ME, Cook S, Leung HY, Neal DE and Robson CN. Expression of Tip60, an androgen receptor coactivator, and its role in prostate cancer development. Oncogene. 2003; 22(16):2466-2477.

44. Hawksworth D, Ravindranath L, Chen Y, Furusato B, Sesterhenn IA, McLeod DG, Srivastava S and Petrovics G. Overexpression of C-MYC oncogene in prostate cancer predicts biochemical recurrence. Prostate cancer and prostatic diseases. 2010; 13(4):311-315.

45. Prochownik EV and Vogt PK. Therapeutic Targeting of Myc. Genes \& cancer. 2010; 1(6):650-659.

46. Kriegl L, Vieth M, Kirchner $\mathrm{T}$ and Menssen A. Upregulation of c-MYC and SIRT1 expression correlates with malignant transformation in the serrated route to colorectal cancer. Oncotarget. 2012; 3(10):1182-1193.

47. Mao B, Zhao G, Lv X, Chen HZ, Xue Z, Yang B, Liu DP and Liang CC. Sirt1 deacetylates c-Myc and promotes c-Myc/Max association. The international journal of biochemistry \& cell biology. 2011; 43(11):1573-1581.

48. Menssen A and Hermeking H. c-MYC and SIRT1 locked in a vicious cycle. Oncotarget. 2012; 3(2):112-113.

49. Menssen A, Hydbring P, Kapelle K, Vervoorts J, Diebold J, Luscher B, Larsson LG and Hermeking H. The c-MYC oncoprotein, the NAMPT enzyme, the SIRT1-inhibitor DBC1, and the SIRT1 deacetylase form a positive feedback loop. Proceedings of the National Academy of Sciences of the United States of America. 2012; 109(4):E187-196.

50. Yuan J, Minter-Dykhouse K and Lou Z. A c-Myc-SIRT1 feedback loop regulates cell growth and transformation. The Journal of cell biology. 2009; 185(2):203-211. 